\title{
Incorporation of Functionalized Multiwall Carbon Nanotubes into a Polyurethane Matrix
}

\author{
Martin Michálek $^{1,2,3}$ and Michael Bredol ${ }^{1}$ \\ ${ }^{1}$ Department of Chemical Engineering, Münster University of Applied Sciences, Stegerwaldstraße 39, 48565 Steinfurt, Germany \\ ${ }^{2}$ Institute of Inorganic Chemistry, Slovak Academy of Sciences, Dubravska cesta 9, 84536 Bratislava 45, Slovakia \\ ${ }^{3}$ Vitrum Laugaricio - Joint Glass Center of the Slovak Academy of Sciences, the Slovak University of Technology, \\ the Alexander Dubček University of Trenčin, and Rona Glassworks, Studentska 2, 91150 Trenčín, Slovakia \\ Correspondence should be addressed to Michael Bredol; bredol@fh-muenster.de
}

Received 16 May 2013; Accepted 17 July 2013

Academic Editor: Jörg M. K. Wiezorek

Copyright @ 2013 M. Michálek and M. Bredol. This is an open access article distributed under the Creative Commons Attribution License, which permits unrestricted use, distribution, and reproduction in any medium, provided the original work is properly cited.

Functionalized and raw multiwall carbon nanotubes (MWCNTs) were investigated colloid-chemically in order to study the role of polar versus nonpolar interaction with a polyurethane (PU) matrix. Both kinds of MWCNTs were dispersed by ultrasonication in the presence of a surfactant (sodium dodecyl sulphate) in aqueous solution. Functional groups on the nanotube surface were characterized by infrared spectroscopy and by the $\zeta$-potential in aqueous suspension. Such suspensions were added to waterborne PU dispersions, drop-cast on glass substrates and cured. The percolation threshold for electrical conductivity with polar (functionalized) MWCNTs was reached at $0.24 \mathrm{wt} . \%$, whereas at concentrations as high as $2 \mathrm{wt. \%}$, PU films with nonpolar MWCNTs stayed below the percolation threshold. With an addition of $0.4 \mathrm{wt} . \%$ polar MWCNTs, the electrical conductivity increased to $>10^{-6} \mathrm{~S} / \mathrm{cm}$ in the cured coating layer. These results are interpreted with respect to the chemical nature of the PU matrix.

\section{Introduction}

The unique combination of mechanical and functional properties makes carbon nanotubes (CNTs) an ideal reinforcing agent for many matrices, including synthetic and natural polymers $[1,2]$. Polymers with CNTs find application in many fields such as corrosion protection $[3,4]$, optically active composites [5], battery technology [6], or chemical sensors [7].

Ideas to use CNTs for charge mitigation in coatings have been around for some time already [8], however, typically for applications like in the aviation industry, where transparency or colour is not rated as high as in domestic applications, and a greyish appearance may be tolerable.

With respect to electrical conductivity, a composite of a polymer with a conductive filler will show reasonable low resistance as soon as the percolation limit is reached; with CNTs of high aspect ratio, this can be the case at considerably smaller volume fraction than with other more spherical conductive fillers. Moreover, there is the possibility to aggregate fillers during the curing process by depletion forces; this approach has been used successfully to produce holographic patterns in polymer nanocomposites [9-11]. Conductivity at drastically smaller CNT contents can also be obtained by a specific texture of the CNTs, as shown in [12], by the use of aligning electrical fields. If similar approaches could be realized with CNTs as well, the volume fraction for reaching percolation should be even smaller than with random distribution and orientation. Of course, such effects will depend crucially on the interface chemistry between polymer and filler, which is the subject of this work.

When preparation or curing of the composites starts in the liquid phase, the preparation of (meta-) stable suspensions of well-separated CNTs is a prerequisite for the fabrication of solid polymer bodies with homogeneously dispersed CNTs. Unfortunately, due to their high aspect ratio, nanosized diameter, and huge surface area, it is very difficult to overcome the strong van der Waals forces between the CNTs to form a stable dispersion. The likely presence of CNT bundles and agglomerates in the final composite can lead to intolerable effects on the desired properties. Despite this 
fact, investigations described in $[13,14]$ showed that a certain amount of agglomerates can decrease the necessary content of CNTs, for obtaining the percolation threshold. To overcome these inherent obstacles and put the excellent mechanical and functional properties of CNTs $[15,16]$ into focus, mechanical treatment and/or chemical functionalization of carbon nanotubes has to be employed; some general approaches to this have been described, for example, in [17].

Mechanical methods available for the incorporation of CNT powders into a liquid (pre-) polymer matrix are stirring processes [18], ball milling [19], and/or ultrasonication [20]. Ultrasonication appears to be the most efficient way for disentangling CNT bundles in the first step of preparation and therefore is commonly used [21]. These methods are called "noncovalent" (the surface chemistry of the CNTs is not altered), and CNTs may be bonded to the polymer through nonpolar types of interaction like $\pi$-stacking, provided that the polymer has conjugated double bonds or heteroatoms with nonbonding electron pairs [22].

Chemical functionalization on the other hand involves replacement of $\mathrm{C}-\mathrm{C}$ bonds on the nanotube surface by functional groups. Functionalizations realized by using acidic and strong oxidant media are convenient methods [23]. With these approaches, covalent attachment of polar functional groups to the CNT surface is obtained. They can interact with amino- or hydroxyl-moieties from polymer chains [16]; therefore dispersion of CNTs in polymers with at least partial polar character becomes easier.

The aim of this work was the preparation of conductive or semiconductive polyurethane (PU)/CNT composites. This class of polymers is very interesting, since it can be prepared in aqueous dispersion, and in this state is the base of a range of waterborne coating formulations, suited also for use under heavy duty, for example, as a finish for wooden floors. The favourable properties of the polymer system under these conditions are at least partially explained by an extended system of $\mathrm{H}$-bridges between the typical polar acid amide units of the polymer, being responsible for the special viscoelastic behaviour of this class of polymer coatings ("reflow, meaning partial self-healing of scratches). Technical PU dispersions typically at the same time are modified by fatty acids; therefore polar as well as nonpolar regions are present in the final films. In view of environmental aspects, nowadays PU dispersions are supplied exclusively as waterborne systems. Applications as, for example, wood finish imply that the final film stays completely transparent and does not change the colour of the underlying substrate too much. Scattering by aggregated fillers or extensive blackish appearance thus will not be tolerable.

However, there is not much information to be found in the literature about composites of such PU dispersions with CNTs; reports available concentrate on the assessment of mechanical properties of the final films and typically use very high loads of CNTs in the blend [24], which will not lead to transparent and colourless coatings. With respect to electrical properties, a recent compilation of 147 polymer composite systems with CNTs lists only two systems with PU matrix, both based on organic solvents [25]. Typical percolation limits over all systems are $>0.2 \mathrm{wt} . \%$, with the exemption of some epoxy-based systems.

In view of the ambipolar character of PU films, this work evaluated nonpolar and polar dispersion methods of CNTs with respect to the homogeneous distribution of multiwall carbon nanotubes (MWCNTs) in a commercially available PU dispersion matrix, ready for use as a wood finish. The results were assessed by the observation of the electrical conductivity in the final material as a proxy for successful dispersion and homogeneous distribution. Results were compared to the development of the optical transmittance in order to correlate a potentially useful increase of the conductivity with (in most cases not wanted) reduced optical transmittance of the films. The effect of this kind of functionalization is discussed in front of the colloid chemical background, and for this purpose, infrared spectra and $\zeta$ potential measurements have been employed.

\section{Materials and Methods}

MWCNTs used in this study are commercially available from Chengdu Organic Chemicals Co., Ltd., China, with purity $>98 \%$. They were synthesized by chemical vapour deposition and have a length of ca. $25 \mu \mathrm{m}$ with a diameter in the range of $7-15 \mathrm{~nm}$. The anionic surfactant sodium dodecyl sulphate (SDS, molecular weight $288.38 \mathrm{~g} \mathrm{~mol}^{-1}$, linear formula $\left.\mathrm{CH}_{3}\left(\mathrm{CH}_{2}\right)_{11} \mathrm{OSO}_{3} \mathrm{Na}\right)$ was supplied by Sigma Aldrich Co., Germany, in order to get a homogeneous dispersion of nanotubes in aqueous solution and to facilitate their incorporation into the polymer matrix. All other chemicals $(\mathrm{NaOH}$, $\mathrm{HCl}, \mathrm{HNO}_{3}$, and polyvinyl alcohol (PVA)) were obtained from Sigma Aldrich Co., Germany, in analytical grade.

Functionalization of raw MWCNTs (r-MWCNTs) was carried out using $65 \%$ concentrated $\mathrm{HNO}_{3}$, at $80^{\circ} \mathrm{C}$ for $2,4,6$, and 8 hours. Treated MWCNTs (t-MWCNTs) were dispersed in $0.1 \mathrm{wt} . \%$ SDS/water solution to obtain a stable suspension by ultrasonication (Sonoplus, HD 3200, MS 73 microneedle, Bandelin electronic GmbH \& Co. KG, 93 W, 45\% amplitude, $10 \mathrm{~min})$.

Prepared CNT-suspension was added to a commercial waterborne $\mathrm{PU}$ dispersion with polyisocyanate hardener (Bona Traffic, a two-component system intended for use as finish of wooden floors, manufacturer: Bona $A B$, Malmö, Sweden) and sonicated for $30 \mathrm{~s}$ at the same conditions as before, with different solid content of t-MWCNTs and rMWCNTs $(0,0.08,0.16,0.24,0.32$, and 0.4 wt.\%). Layers on glass substrates were formed by a simple casting technique of the suspension with an amount of $0.3 \mathrm{~mL}$ on an area of $55 \times 20 \mathrm{~mm}$.

Fourier transform infrared spectroscopy (FTIR) was used before and after functionalization of the MWCNTs. For this purpose, the KBr disk technique was utilized (Perkin Elmer, Spectrum 100 FT-IR Spectrometer).

A Zetasizer Nano (Malvern Instruments Ltd., UK) was employed for measuring the $\zeta$-potential of raw and treated particles in aqueous suspension. For these measurements, the suspensions were diluted to a concentration of $0.1 \mathrm{wt} . \%$, and the $\mathrm{pH}$ was shifted with $0.5 \mathrm{M} \mathrm{HCl}$ and $0.1 \mathrm{M} \mathrm{NaOH}$. Ultrasonication was carried out at $93 \mathrm{~W}$ and $45 \%$ amplitude for 
10 min prior to measurements. From repeated messurements on calibration samples, the uncertainty of an individual measurement is estimated to be $\pm 2 \mathrm{mV}$.

Optical transmittance was measured with a Thermo Scientific Genesys 10S Series ultraviolet-visible spectrophotometer.

The specific electrical resistivity of films was measured with a Mastech Digital Multimeter MS8229 (Pollin Electronic). Measuring was carried out on the layers prepared on a glass substrate, with an electrode distance of $1 \mathrm{~cm}$. Values of electrical resistivity $>10^{8} \Omega \mathrm{cm}$ are out of the range of this device and have not been processed further.

\section{Results and Discussion}

In preparation of the fabrication of composites, the asreceived MWCNTs were first functionalized in hot concentrated $\mathrm{HNO}_{3}$ for 2, 4, 6, and 8 hours. One of the indicators for successful treatment is the presence of functional groups as probed by FTIR spectroscopy; the development of such spectra over time of functionalization was used to find conditions for optimal pretreatment.

The conditions used for functionalization caused small changes in band intensities after 2 and 4 hours, while after 6 hours, the full formation of a new band system centered at $1635 \mathrm{~cm}^{-1}$ was observed, which can be assigned to a water bending mode (also present in t-MWCNTs). This effect however might well be caused by humidity adsorbed from the environment during the measurement procedure and thus is visible also on the unprocessed raw material. More interesting, the generated nonpolar groups $(\mathrm{C}-\mathrm{H}$ bond stretching at $2922.95 \mathrm{~cm}^{-1}$ and $2852.53 \mathrm{~cm}^{-1}$ ) at the MWCNT surface are clearly visible only after $8 \mathrm{~h}$ of functionalization in $\mathrm{HNO}_{3}$. Therefore in the following, only composites with tMWCNTs having received $8 \mathrm{~h}$ of functionalization in $\mathrm{HNO}_{3}$ will be discussed in more detail. Functional groups like $-\mathrm{COOH},-\mathrm{C}-\mathrm{O}-$, or $\mathrm{C}=\mathrm{O}$ were not observed in the FTIR spectra, probably due to their low concentration, although they should be present under the strong oxidizing conditions of functionalization. This is in accordance with the investigations described in [26], where among the functional groups introduced by functionalization, $\mathrm{CH}_{x}$ were dominating (76.6\%); O-containing entities made up for less than $25 \%$ of the groups introduced.

In order to assess the polar surface groups after functionalization more directly, $\zeta$-potential measurements in aqueous dispersion were performed and are depicted in Figure 1. Only the $\mathrm{O}$-containing surface groups are polar enough to either dissociate or accept a proton from solution; changes in the curves of $\zeta$-potential over $\mathrm{pH}$ thus are coupled directly with the presence of polar groups on the nanotube surface. It was found that the functionalization with $\mathrm{HNO}_{3}$ has a great impact on the surface charge. The isoelectric point of $r-$ MWCNTs (close to $\mathrm{pH}=8$ ) is strongly shifted to the acidic region (ca. $\mathrm{pH}=2$ ) in case of t-MWCNTs. Obviously, the polar surface groups invisible in the IR spectra, due to their comparatively low concentration, dominate the interface chemistry of the CNTs. Although this effect is present after short functionalization time already, there is a clear

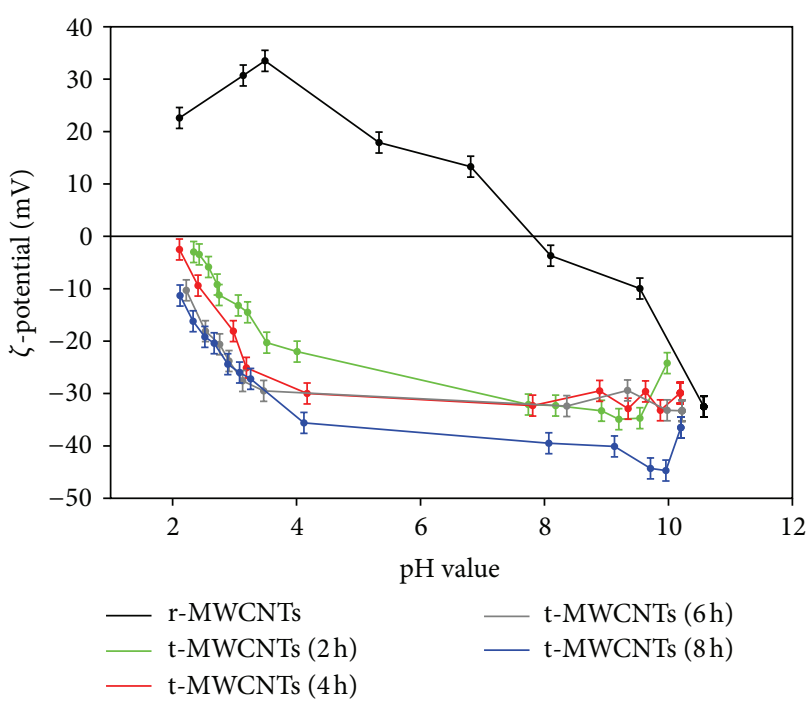

FIGURE 1: $\zeta$-potential of r-MWCNTs and t-MWCNTs as function of $\mathrm{pH}$ value and treatment time. Lines are guidelines for the eye only.

trend for still decreasing $\zeta$-potential at prolonged functionalization. (Anionic) SDS alone on r-MWCNTs does establish a negative surface potential at high $\mathrm{pH}$ range only, since the only way of interaction is of weak nonpolar nature. After functionalization of the MWCNTs, SDS adsorbs very strongly by polar interaction, establishing a negative $\zeta$-potential in the whole $\mathrm{pH}$ range investigated (down to $\mathrm{pH}=2$, which is close to the $\mathrm{pKs}$ value of sulfonic acids). Taking the amphiphile SDS as a proxy for ambipolar PU, the polar interaction clearly is much stronger than the nonpolar one, and it is to be expected that $\mathrm{t}-\mathrm{MWCNT}$ can be dispersed much better in aqueous PU dispersion as well as in the PU matrix as it develops during curing.

Due to functionalization, the electrostatic repulsion between the t-MWCNTs is obviously increased and causes more stable suspensions in a wide range of $\mathrm{pH}$ values. The maximum absolute value of the $\zeta$-potential was reached with

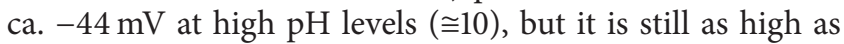
$-35 \mathrm{mV}$ at $\mathrm{pH}=4$. The strong (stabilizing) charge at the t-MWCNT surface therefore can be retained over the full (technically) useful $\mathrm{pH}$ range. However, t-MWCNTs with adsorbed SDS do offer polar as well as some nonpolar surface regions, therefore being able to interact with both parts of the PU network.

Scanning electron microscopy (SEM) micrographs of $\mathrm{r}$ MWCNTs and t-MWCNTs after $8 \mathrm{~h}$ are depicted in Figures 2(a) and 2(b). r-MWCNTs form bundles, while t-MWCNTs after dispersion in SDS solution and subsequent drying are well separated. For comparison, a solution using polyvinyl alcohol (PVA, predominantly polar interaction) as dispersant was prepared in the same way. In Figure 2(c), agglomerates of MWCNTs are clearly visible in a transmission electron microscopy (TEM) micrograph, with diameters $>1 \mu \mathrm{m}$. Here, interaction is too strong and leads to untolerable agglomeration tendencies. 


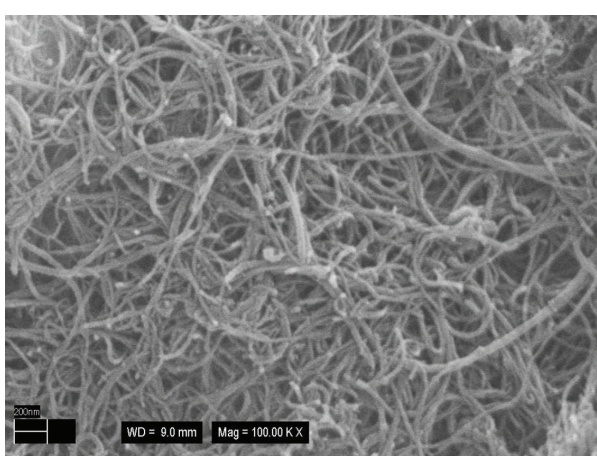

(a)

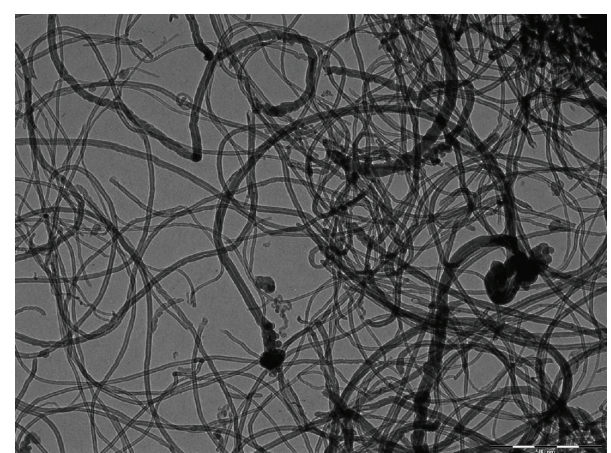

(b)

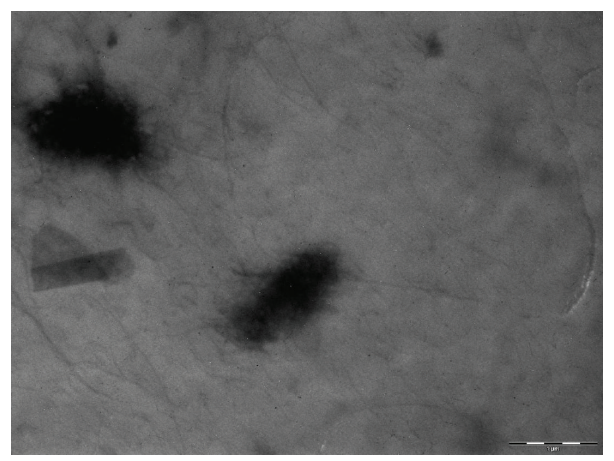

(c)

FIGURE 2: SEM micrographs of r-MWCNTs ((a) scalebar $200 \mathrm{~nm}$ ), t-MWCNTs in SDS solution ((b) scalebar $500 \mathrm{~nm})$, TEM micrograph of $\mathrm{t}-\mathrm{MWCNTs}$ in PVA solution ((c) scalebar $1 \mu \mathrm{m})$.

Unfavourable agglomeration effects on the distribution of MWCNTs will also influence the electrical conductivity of the material. Electrical conductivity in this case was not reached even with MWCNT content of $>2$ vol\%. Therefore, further experiments were performed with MWCNTs dispersed in SDS solution only.

A direct comparison between incorporation of $\mathrm{t}$ MWCNTs and r-MWCNTs into the waterborne PU dispersion is depicted in Figure 3, from the point of view of visible appearance.

More polar surface nature of t-MWCNTs leads to more homogenous dispersion of these CNTs in the composite as compared to the noncovalent bonding. SDS from aqueous solution is attached to the functional groups of the tMWCNTs surface and inhibits the van der Waals attractive forces, reducing the formation of bundles. Nonpolar interaction alone between the untreated nanotube surface and SDS does not lead to homogeneous distribution in the polymer matrix. The nonpolar nature of r-MWCNTs leads to unprotected particles and thus to the creation of agglomerates, optically visible even with the naked eye from the lowest solid loading of $0.08 \mathrm{wt} . \%$ and then to an increasing amount of bundles with increasing amounts of loaded r-MWCNTs.

Electrical conductivity in cured coatings of $>10^{-6} \mathrm{~S} \mathrm{~cm}^{-1}$ assures dissipation of free electrostatic charges and thus is a prerequisite, when electrostatic charging needs to be avoided. A plot of electrical conductivity over concentration of the prepared coating films is shown in Figure 4. At ca. 0.24 wt.\% of $\mathrm{t}$-MWCNTs the percolation threshold for conductivity was observed, whereas at approximately $0.32 \mathrm{wt} . \%$ solid content of $\mathrm{t}$-MWCNTs the required level of electrical conductivity is reached.

Comparing these results to the entries in the compilation in [25] shows that the covalent functionalization with polar groups leads to state-of-the-art dispersions, whereas without that functionalization, heavy agglomeration is induced, leading to lower conductivities and higher percolation thresholds. For nanocomposites of MWCNTs with PU, the message is clear and has been underpinned by the data compiled in this work: full dispersion can only be reached when the filler (here: MWCNTs) is functionalized with polar groups in order to keep it compatible with the more polar parts of the polymer as well as with the nonpolar parts. Without polar functionlization, no composites can be formed by simple mixing processes.

Unfortunately, high electrical conductivity correlates with low optical transmittance, as compared to the pure material. From Figure 5, it is obvious that the effect on transmittance is enormous. At threshold for electrical conductivity, the transmittance has dropped in the optical region to ca. $10 \%$ of the pure polymer, which will not be acceptable for most optical applications.

\section{Conclusions}

Functionalization of MWCNTs with $65 \% \mathrm{HNO}_{3}$ for 8 hours induced enough polar bonding sites on the surface of the nanotubes to achieve well dispersed aqueous suspensions, 


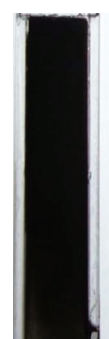

0.4

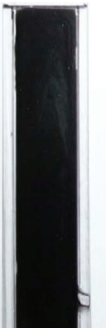

0.32

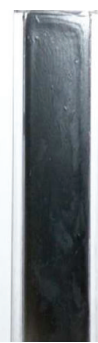

0.24

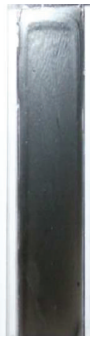

0.16

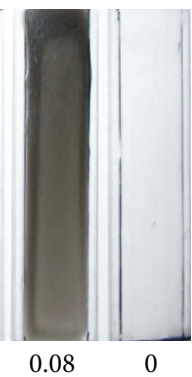

(a)
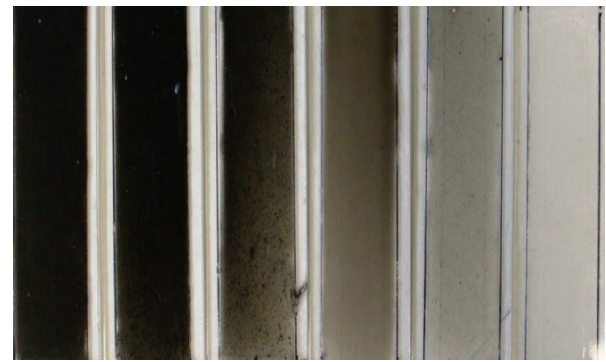

(b)

Figure 3: Photographs of coatings prepared with t-MWCNTs (a) and r-MWCNTS (b) at various loading levels.

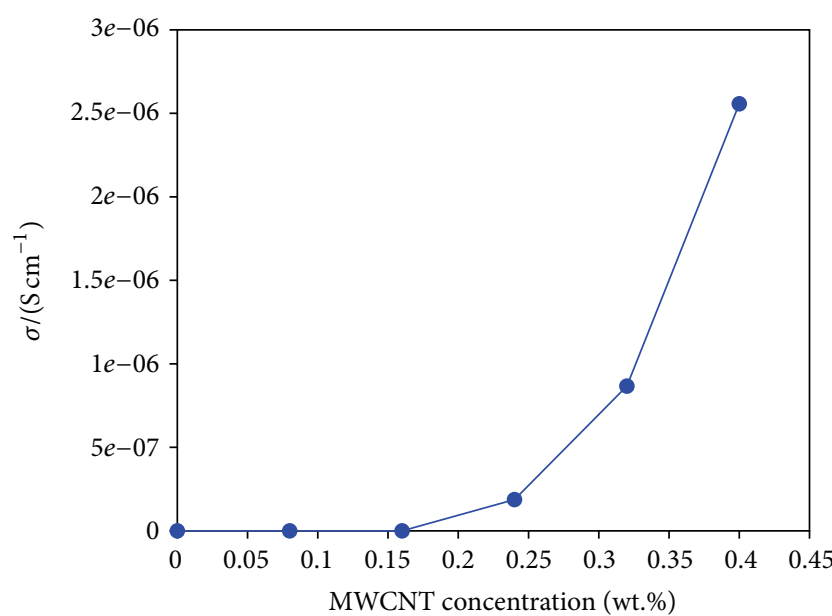

FIGURE 4: Electrical conductivity of coating films prepared; the line is a guideline for the eye.

miscible with waterborne PU dispersions by simple techniques (stirring and ultrasonication). The presence of polar surface groups was confirmed by $\zeta$-potential measurements in suspension; negative surface charge then was observed over the full range of $\mathrm{pH}$ values investigated. After curing, the MWCNTs were distributed homogeneously in the polymer matrix, as judged by the percolation threshold of conductivity. The functionalized MWCNTs therefore were not only well dispersed, but also readily accepted by the ambipolar PU matrix during the coating as well as the curing process. It was possible to obtain an electrical conductivity $>10^{-6} \mathrm{~S} \mathrm{~cm}^{-1}$ with $0.4 \mathrm{wt} . \%$ of polar MWCNTs in the PU

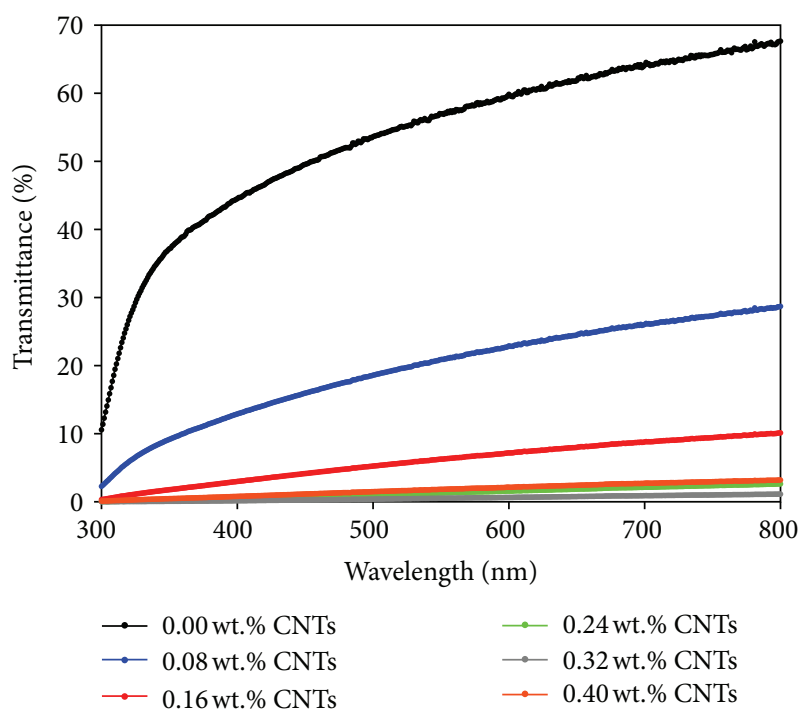

FIGURE 5: Optical transmittance of coating films prepared; the line is a guideline for the eye.

matrix. However, the optical transmittance of the conductive films was so small that unacceptable decolorization would occur in coating applications. Therefore, the use of MWCNTs as charge mitigator in PU in transparent coating systems does not only need functionalization of the nanotubes with polar groups, but rather additional alignment or texturizing to achieve conductivity simultaneously with sufficiently high transparency.

\section{Acknowledgments}

Parts of this work were supported by a grant of the HeinrichHertz-foundation of the Federal State of North RhineWestphalia, Germany. The project was initiated by K.-H. Bode of Bode Parkett, Geldern, Germany. Inspiring discussions with L. Sallinger of IRSA Lackfabrik, Deisenhausen, Germany were much appreciated.

\section{References}

[1] J. N. Coleman, "Liquid-phase exfoliation of nanotubes and graphene ," Advanced Functional Materials, vol. 19, no. 23, pp. 3680-3695, 2009.

[2] K. Wei, J.-H. Xia, B.-S. Kim, and I.-S. Kim, "Multiwalled carbon nanotubes incorporated bombyx mori silk nanofibers by electrospinning," Journal of Polymer Research, vol. 18, no. 4, pp. 579-585, 2011.

[3] C. B. Breslin, A. M. Fenelon, and K. G. Conroy, "Surface engineering: corrosion protection using conducting polymers," Materials and Design, vol. 26, no. 3, pp. 233-237, 2005.

[4] P. Herrasti, F. Recio, P. Ocón, and E. Fatás, "Effect of the polymer layers and bilayers on the corrosion behaviour of mild steel: comparison with polymers containing Zn microparticles," Progress in Organic Coatings, vol. 54, no. 4, pp. 285-291, 2005.

[5] H. S. Woo, R. Czerw, S. Webster et al., "Hole blocking in carbon nanotube-polymer composite organic light-emitting 
diodes based on poly (m-phenylene vinylene-co-2, 5-dioctoxyp-phenylene vinylene)," Applied Physics Letters, vol. 77, no. 9, pp. 1393-1395, 2000.

[6] A. Mirmohseni and R. Solhjo, "Preparation and characterization of aqueous polyaniline battery using a modified polyaniline electrode," European Polymer Journal, vol. 39, no. 2, pp. 219-223, 2003.

[7] J. Kong, N. R. Franklin, C. Zhou et al., "Nanotube molecular wires as chemical sensors," Science, vol. 287, no. 5453, pp. 622625, 2000.

[8] K. A. Watson, S. Ghose, D. M. Delozier, J. G. Smith Jr., and J. W. Connell, "Transparent, flexible, conductive carbon nanotube coatings for electrostatic charge mitigation," Polymer, vol. 46, no. 7, pp. 2076-2085, 2005.

[9] T. Ninjbadgar, G. Garnweitner, A. Börger, L. M. Goldenberg, O. V. Sakhno, and J. Stumpe, "Synthesis of luminescent $\mathrm{ZrO}_{2}: \mathrm{Eu}^{3+}$ nanoparticles and their holographic sub-micrometer patterning in polymer composites," Advanced Functional Materials, vol. 19, no. 11, pp. 1819-1825, 2009.

[10] T. N. Smirnova, O. V. Sakhno, P. V. Yezhov, L. M. Kokhtych, L. M. Goldenberg, and J. Stumpe, "Amplified spontaneous emission in polymer-CdSe/ZnS-nanocrystal DFB structures produced by the holographic method," Nanotechnology, vol. 20, no. 24, Article ID 245707, 2009.

[11] O. V. Sakhno, T. N. Smirnova, L. M. Goldenberg, and J. Stumpe, "Holographic patterning of luminescent photopolymer nanocomposites," Materials Science and Engineering C, vol. 28, no. 1, pp. 28-35, 2008.

[12] C. A. Martin, J. K. W. Sandler, A. H. Windle et al., "Electric fieldinduced aligned multi-wall carbon nanotube networks in epoxy composites," Polymer, vol. 46, no. 3, pp. 877-886, 2005.

[13] J. Aguilar, J. Bautista-Quijano, and F. Avilés, "Influence of carbon nanotube clustering on the electrical conductivity of polymer composite films," eXPRESS Polymer Letters, vol. 4, no. 5, pp. 292-299, 2010.

[14] J. J. Hernández, M. C. García-Gutiérrez, A. Nogales et al., "Influence of preparation procedure on the conductivity and transparency of SWCNT-polymer nanocomposites," Composites Science and Technology, vol. 69, no. 11-12, pp. 1867-1872, 2009.

[15] E. T. Thostenson, Z. Ren, and T.-W. Chou, "Advances in the science and technology of carbon nanotubes and their composites: a review," Composites Science and Technology, vol. 61, no. 13, pp. 1899-1912, 2001.

[16] D. Tasis, N. Tagmatarchis, A. Bianco, and M. Prato, "Chemistry of carbon nanotubes," Chemical Reviews, vol. 106, no. 3, pp. 1105-1136, 2006.

[17] M. Bradley, "Chemistry at the polymer-particle interface for the design of innovative materials," Soft Matter, vol. 8, no. 5, pp. 1268-1274, 2012.

[18] B. P. Grady, F. Pompeo, R. L. Shambaugh, and D. E. Resasco, "Nucleation of polypropylene crystallization by single-walled carbon nanotubes," Journal of Physical Chemistry B, vol. 106, no. 23, pp. 5852-5858, 2002.

[19] G. Gorrasi, M. Sarno, A. Di Bartolomeo, D. Sannino, P. Ciambelli, and V. Vittoria, "Incorporation of carbon nanotubes into polyethylene by high energy ball milling: morphology and physical properties," Journal of Polymer Science B, vol. 45, no. 5 , pp. 597-606, 2007.

[20] B. Kim, J. Lee, and I. Yu, "Electrical properties of single-wall carbon nanotube and epoxy composites," Journal of Applied Physics, vol. 94, no. 10, pp. 6724-6728, 2003.
[21] H. Bang, M. Gopiraman, B. S. Kim, S. H. Kim, and I. S. Kim, "Effects of pH on electrospun PVA/acid-treated MWNT composite nanofibers ," Colloids and Surfaces A, vol. 409, pp. 112-117, 2012.

[22] Z. Spitalsky, D. Tasis, K. Papagelis, and C. Galiotis, "Carbon nanotube-polymer composites: chemistry, processing, mechanical and electrical properties," Progress in Polymer Science, vol. 35, no. 3, pp. 357-401, 2010.

[23] S. Ramesh, L. M. Ericson, V. A. Davis et al., "Dissolution of pristine single walled carbon nanotubes in superacids by direct protonation," Journal of Physical Chemistry B, vol. 108, no. 26, pp. 8794-8798, 2004.

[24] J. Kwon and H. Kim, "Comparison of the properties of waterborne polyurethane/multiwalled carbon nanotube and acidtreated multiwalled carbon nanotube composites prepared by in situ polymerization," Journal of Polymer Science A, vol. 43, no. 17, pp. 3973-3985, 2005.

[25] W. Bauhofer and J. Z. Kovacs, "A review and analysis of electrical percolation in carbon nanotube polymer composites," Composites Science and Technology, vol. 69, no. 10, pp. 14861498, 2009.

[26] L. Liu, Y. Qin, Z.-X. Guo, and D. Zhu, "Reduction of solubilized multi-walled carbon nanotubes," Carbon, vol. 41, no. 2, pp. 331$335,2003$. 

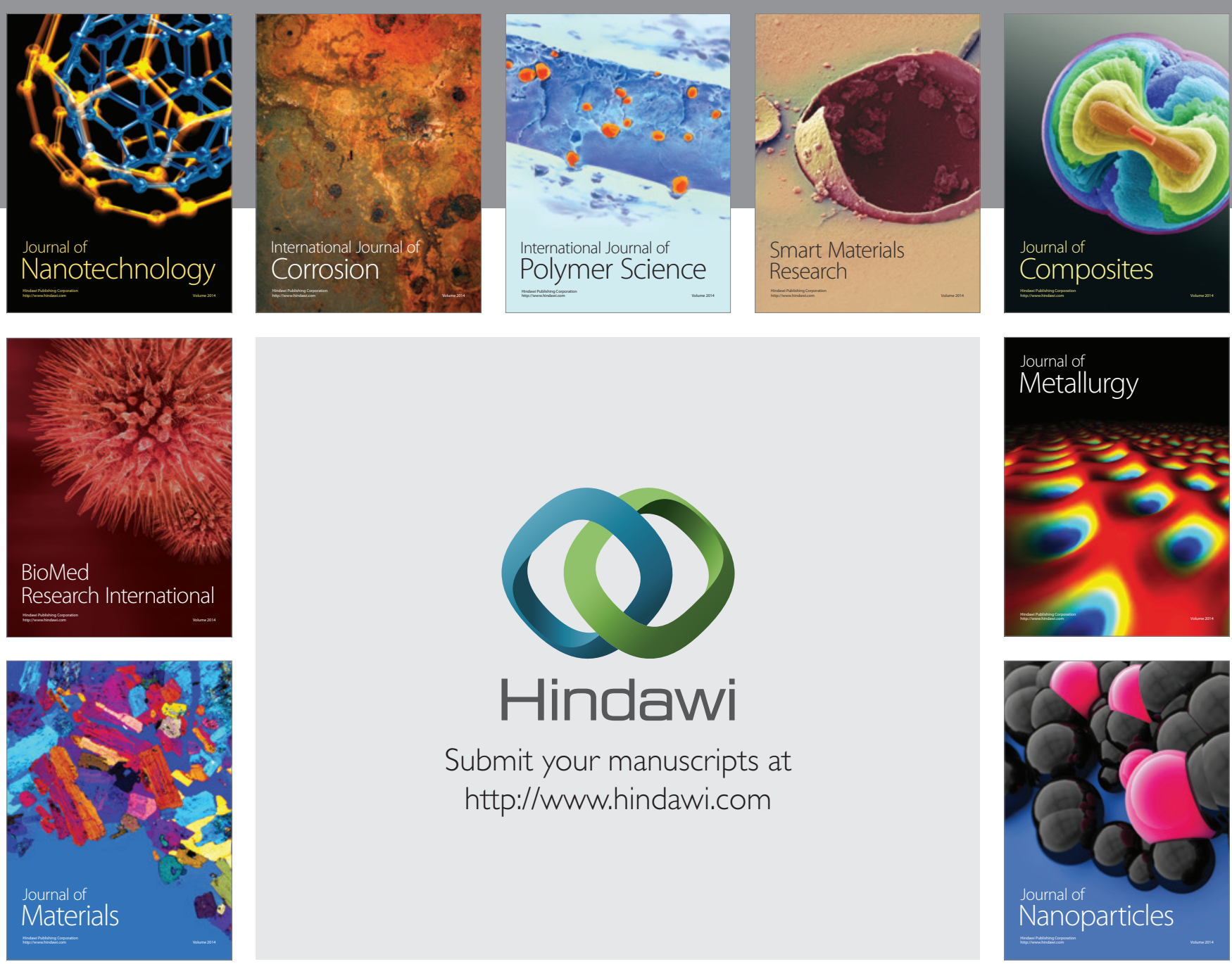

Submit your manuscripts at http://www.hindawi.com
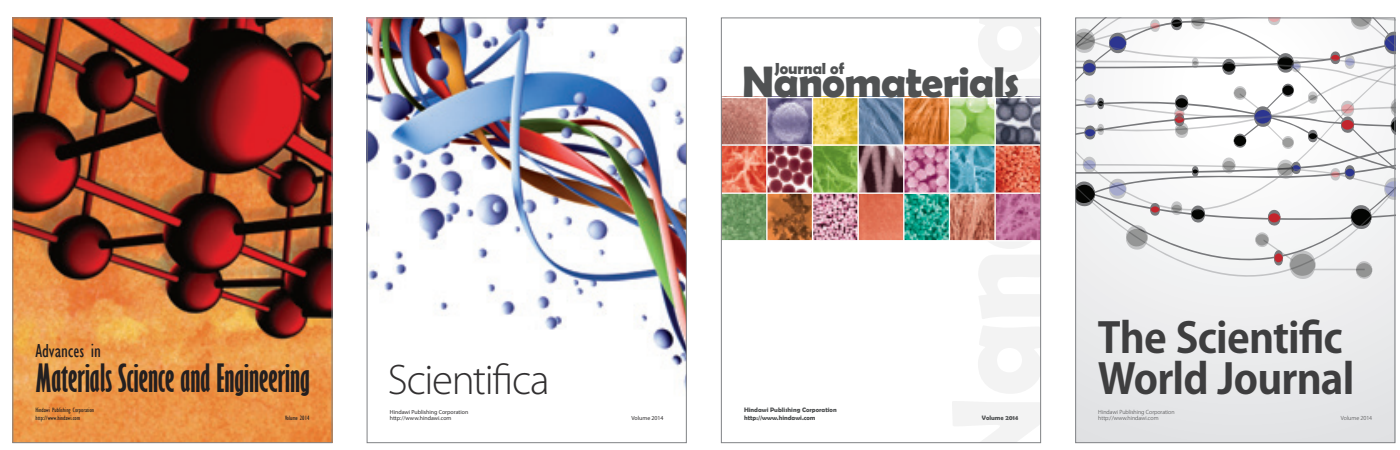

\section{The Scientific World Journal}
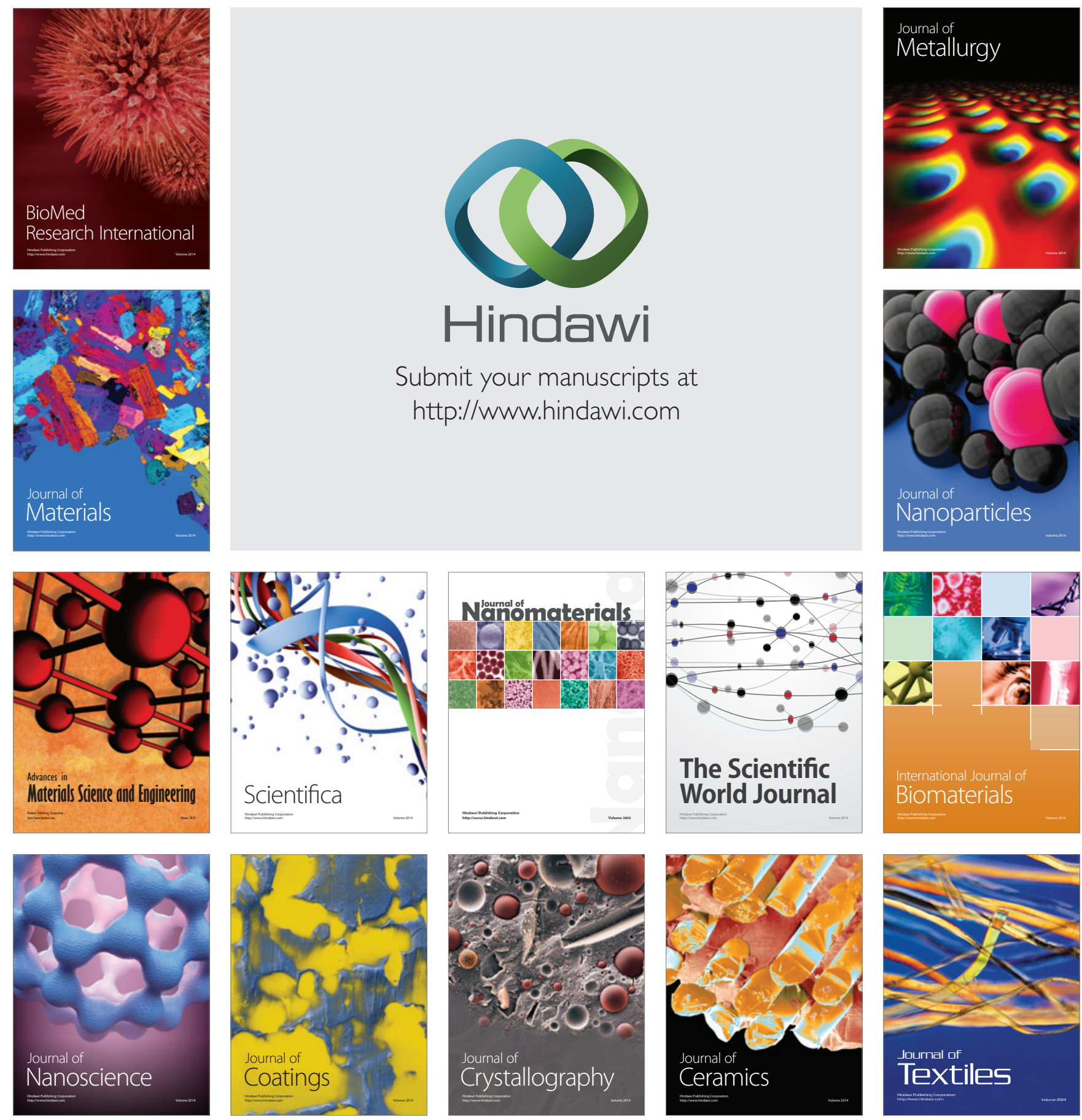